\title{
Heterogeneous perturbation of fluid density and solid elastic strain in consolidating porous media
}

\author{
P. Artale Harris \\ Dipartimento di Scienze di Base e Applicate per l'Ingegneria, Sapienza Università di Roma, via \\ A. Scarpa 16, I-00161, Roma, Italy. \\ E_mail: pietro.artale.h@gmail.com \\ E.N.M. Cirillo \\ Dipartimento di Scienze di Base e Applicate per l'Ingegneria, Sapienza Università di Roma, via \\ A. Scarpa 16, I-00161, Roma, Italy. \\ E_mail: emilio.cirillo@uniroma1.it \\ G. Sciarra \\ Dipartimento di Ingegneria Chimica Materiali Ambiente, Sapienza Università di Roma, via \\ Eudossiana 18, 00184 Roma, Italy \\ E_mail: giulio.sciarra@uniroma1.it
}

\begin{abstract}
The occurrence of heterogeneous perturbations of fluid mass density and solid elastic strain of a porous continuum, as a consequence of its undrained response is a very important topic in theoretical and applied poromechanics. The classical MandelCryer effect provides an explanation of fluid overpressure in the central region of a porous sample, immediately after the application of the loading. However this effect fades away when the fluid leaks out of the porous network. Here this problem is studied within the framework of a second gradient theory and a thorough description of the static and the dynamics of the phenomenon is given.
\end{abstract}

\section{Introduction}

When a mechanical pressure is exherted on the solid skeleton of a porous medium and its elastic strain is a consequence of the variation of the fluid mass content inside the pores, several interesting phenomena can occur which accompany shrinkage or swelling of the solid skeleton. In this paper attention is focused on the occurrence of heterogeneous perturbations of the fluid mass density and the skeleton elastic strains, as a consequence of the undrained response of the porous medium. The classical MandelCryer effect, see 17] and 12, provides an explanation, within multidimensional consolidation, of fluid overpressure, in the central region of the sample, immediately after the application of the loading. However, in that case this is a definitely non-permanent effect which fades away when the fluid leaks out the boundary and the pore pressure reverses and dissipates. The physical background of the Mandel-Cryer effect is that the generation of fluid over-pressure due to loading is immediate, but the dissipation due to the fluid flow is retarded by the permeability and the distance to the drainage boundary. On the other hand several authors discussed the onset of strain localization during globally undrained triaxial tests, in particular for loose granular materials, see e.g., [19], [18], or [24]. In this case local fluid exchange is allowed, even in presence of localized strain, inside the specimen until, at high level of confinement, the pore pressure generation inside the band leads locally to fluidization of the crushed material, which results into the formation of connected channels in the heart of the 
band. Similar confinement effects have also been recorded in a fluidized column test, see [20], where a fluid is forced to flow through a saturated sample from the bottom. By tuning the velocity of the fluid, the drag force acting on the solid grains, possibly causing unbalance of gravity force, is controlled [25,26]. These experimental results demonstrate that under consolidation loading, and because of porosity change, the fluid can migrate through the pores and eventually remain segregated, possibly enhancing localized overpressurization and fluidization of the soil, see e.g. [16, 20].

In the papers [6 8] and [10] we have attacked this problem from the point of view of bifurcation theory and we have shown that it is possible to describe interesting phenomena (still in the range of non-linear elasticity) taking place when the confining pressure exerted on the solid exceeds a suitable limiting value. The idea is to get a formulation capable for describing the onset of a fluid-rich and a fluid-poor phase, eventually coexisting inside the porous skeleton at equilibrium. Introducing a nonlocal energy contribution, which penalizes gradients of strain and fluid mass density, a smooth transition between phases of the porous medium, associated with different fluid content, has been modeled, so accounting for the arising of the above mentioned heterogeneous elastic strains. Undrained conditions are therefore locally achieved where fluid segregation is attained, even if a standard Darcean dissipative process, associated to the fluid flowing out of the drainage boundary, occurs.

Assuming the potential energy to be quadratic in the first derivatives of the strain and of the fluid mass density variation, the evolution is described by a Cahn-Hilliardlike equation provided that the dissipative forces are proportional to the seepage velocity, say the velocity of the fluid with respect to the solid. This means that the above mentioned assumption of Darcean flow still remains valid.

Within this modeling framework, a generalized consolidation problem for a onedimensional porous continuum is analyzed so extending the classical results due to Terzaghi and those ones, relative to a gradient model, previously obtained by one of the authors, and co-workers 22], in which only the fluid-poor phase was admissible at equilibrium. The equation governing the behavior of the fluid constituent is of higher (fourth) order with respect to the Laplace equation which classically prescribes the behavior of the pore-water pressure. Following previous results reported in [10], different boundary conditions can be considered, in particular essential or natural boundary conditions relative to the velocity of the fluid relative to the solid the fluid chemical potential as well as to the fluid mass density or its spatial gradient; where the dependence of the boundary value problem on higher order derivatives has been taken into account. Here we shall address the two cases in which zero chemical potential, see section 2.4, or zero fluid velocity, say impermeability of the porous skeleton, see section 2.5. have been assumed on the whole or part of the boundary, together with essential boundary conditions on the strain of the solid and the density of the fluid. As already mentioned the interest will be in the occurrence of heterogeneous elastic strains of the solid skeleton and variations of the fluid density; the confining pressure is therefore chosen so as to guarantee the coexistence of phases and, consequently, the onset and the propagation, up to its stationary placement, of the interface between them, see [9].

\section{The model}


We introduce the one dimensional poromechanical model [10] whose geometrically linearized version will be studied in the following sections. Kinematics will be briefly resumed starting from the general statement of the model [11] together with some particular issue introduced in [22]. The equations governing the behavior of the porous system will then be deduced prescribing the conservative part of the constitutive law through a suitable potential energy density $\Phi$ and the dissipative contributions through purely Darcy terms.

\subsection{Poromechanics setup}

Let $B_{\mathrm{s}}:=\left[\ell_{1}, \ell_{2}\right] \subset \mathbb{R}$, with $\ell_{1}, \ell_{2} \in \mathbb{R}$, and $B_{\mathrm{f}}:=\mathbb{R}$ be the reference configurations for the solid and fluid components $\left[11\right.$. The solid placement $\chi_{\mathrm{s}}: B_{\mathrm{s}} \times \mathbb{R} \rightarrow \mathbb{R}$ is a $C^{2}$ function such that the map $\chi_{\mathrm{s}}(\cdot, t)$, associating to each $X_{\mathrm{s}} \in B_{\mathrm{s}}$ the position occupied at time $t$ by the particle labeled by $X_{\mathrm{s}}$ in the reference configuration $B_{\mathrm{s}}$, is a $C^{2}$ diffeomorphism. The fluid placement map $\chi_{\mathrm{f}}: B_{\mathrm{f}} \times \mathbb{R} \rightarrow \mathbb{R}$ is defined analogously. The current configuration $B_{t}:=\chi_{\mathrm{s}}\left(B_{\mathrm{s}}, t\right)$ at time $t$ is the set of positions of the superposed solid and fluid particles.

Consider the $C^{2}$ function $\phi: B_{\mathrm{s}} \times \mathbb{R} \rightarrow B_{\mathrm{f}}$ such that $\phi\left(X_{\mathrm{s}}, t\right)$ is the fluid particle that at time $t$ occupies the same position of the solid particle $X_{\mathrm{s}}$; assume, also, that $\phi(\cdot, t)$ is a $C^{2}$-diffeomorphism mapping univocally a solid particle into a fluid one. The three fields $\rfloor^{1} \chi_{\mathrm{s}}, \chi_{\mathrm{f}}$, and $\phi$ are not at all independent; indeed, by definition, we immediately have that $\chi_{\mathrm{f}}\left(\phi\left(X_{\mathrm{s}}, t\right), t\right)=\chi_{\mathrm{s}}\left(X_{\mathrm{s}}, t\right)$ for any $X_{\mathrm{s}} \in B_{\mathrm{s}}$ and $t \in \mathbb{R}$.

The Lagrangian velocities are two maps associating with each time and each point in the solid and fluid reference space the velocities of the corresponding solid and fluid particles at the specified time. More precisely, the Lagrangian velocities are the two maps $u_{\alpha}: B_{\alpha} \times \mathbb{R} \rightarrow \mathbb{R}$ defined by setting

$$
u_{\alpha}\left(X_{\alpha}, t\right):=\frac{\partial \chi_{\alpha}}{\partial t}\left(X_{\alpha}, t\right)
$$

for any $X_{\alpha} \in B_{\alpha}$, where $\alpha=\mathrm{s}$, f. We also consider the Eulerian velocities $v_{\alpha}: B_{t} \times$ $\mathbb{R} \rightarrow \mathbb{R}$ associating with each point $x \in B_{t}$ and for each time $t \in \mathbb{R}$ the velocities of the solid and fluid particle occupying the place $x$ at time $t$; more precisely we set $v_{\alpha}(x, t):=u_{\alpha}\left(\chi_{\alpha}^{-1}(x, t), t\right)$.

In studying the dynamics of the porous system one can arbitrarily choose two among the three fields $\chi_{\mathrm{s}}, \chi_{\mathrm{f}}$, and $\phi$. Since the reference configuration $B_{\mathrm{s}}$ of the solid component is know a priori, a good choice appears to be that of expressing all the dynamical observables in terms of the fields $\chi_{\mathrm{s}}$ and $\phi$ which are defined on $B_{\mathrm{s}}$. Consider the Lagrangian velocity $u_{\mathrm{f}}$ of the fluid component which is defined on $B_{\mathrm{f}} \times \mathbb{R}$; we prove that for any $X_{\mathrm{s}} \in B_{\mathrm{s}}$ and $t \in \mathbb{R}$

$$
u_{\mathrm{f}}\left(\phi\left(X_{\mathrm{s}}, t\right), t\right)=u_{\mathrm{s}}\left(X_{\mathrm{s}}, t\right)-\frac{\chi_{\mathrm{s}}^{\prime}\left(X_{\mathrm{s}}, t\right)}{\phi^{\prime}\left(X_{\mathrm{s}}, t\right)} \dot{\phi}\left(X_{\mathrm{s}}, t\right)
$$

which means that the velocity of the fluid relative to the solid $v\left(X_{\mathrm{s}}, t\right):=u_{\mathrm{f}}\left(\phi\left(X_{\mathrm{s}}, t\right), t\right)-$ $u_{\mathrm{s}}\left(X_{\mathrm{s}}, t\right)$, in the following seepage velocity, is proportional to the opposite of $\dot{\phi}\left(X_{\mathrm{s}}, t\right)$.

\footnotetext{
${ }^{1}$ In the sequel we shall often use the inverse functions of the space sections of the field $\chi_{\mathrm{f}}, \chi_{\mathrm{s}}$, and $\phi$. We shall misuse the notation and let $\phi^{-1}(\cdot, t)$ be the inverse of the map $X_{\mathrm{s}} \rightarrow \phi\left(X_{\mathrm{s}}, t\right)$ at a given time $t$. Similarly we shall also consider $\chi_{\mathrm{s}}^{-1}(\cdot, t)$ and $\chi_{\mathrm{f}}^{-1}(\cdot, t)$.
} 
The dot and the prime denote, here and in the sequel, the partial derivative with respect to time and to the space variable $X_{\mathrm{s}}$ respectively.

\subsection{Equations of motion}

In [10] we have derived the equations of motion by using a variational approach much similar to that developed in [22]. We shall recall, here, the main steps in the computation.

It is natural to assume that, if the system is acted upon only by conservative forces, its dynamics is described by a Lagrangian density $\mathscr{L}$, relative to the solid reference configuration space volume, depending on the space variable $X_{\mathrm{s}}$ and on time through (in principle) $\dot{\chi}_{\mathrm{s}}, \dot{\phi}, \chi_{\mathrm{s}}^{\prime \prime}, \phi^{\prime \prime}, \chi_{\mathrm{s}}^{\prime}, \phi^{\prime}, \chi_{\mathrm{s}}$, and $\phi$. The Lagrangian density is equal to the kinetic energy density minus the overall potential energy density accounting for both the internal and the external conservative forces. The equations of motion for the two fields $\chi_{\mathrm{s}}$ and $\phi$ can be derived assuming that the possible motions of the system in an interval of time $\left(t_{1}, t_{2}\right) \subset \mathbb{R}$ are those such that the fields $\chi_{\mathrm{s}}$ and $\phi$ are extremals for the action functional

$$
A\left(\chi_{\mathrm{s}}, \phi\right):=\int_{t_{1}}^{t_{2}} \mathrm{~d} t \int_{B_{\mathrm{s}}} \mathrm{d} X_{\mathrm{s}} \mathscr{L}\left(\dot{\chi}_{\mathrm{s}}\left(X_{\mathrm{s}}, t\right), \ldots, \phi\left(X_{\mathrm{s}}, t\right)\right)
$$

in correspondence of the independent variations of the two fields $\chi_{\mathrm{s}}$ and $\phi$ on $B_{\mathrm{s}} \times\left(t_{1}, t_{2}\right)$. In other words any possible motion of the system in the considered interval is a solution of the Euler-Lagrange equations associated to the variational principle $\delta A=0$.

A different variational principle is needed if the fluid component of the system is acted upon by dissipative forces; the virtual work made by these forces must be taken into account. Consider the independent variations $\delta \chi_{\mathrm{s}}$ and $\delta \phi$ of the two fields $\chi_{\mathrm{s}}$ and $\phi$ and denote by $\delta W$ the corresponding elementary virtual work made by the dissipative forces acting on the fluid component. The possible motions of the system, see for instance [5, Chapter 5], in an interval of time $\left(t_{1}, t_{2}\right) \subset \mathbb{R}$ are those such that the fields $\chi_{\mathrm{s}}$ and $\phi$ satisfies the variational principle

$$
\delta A=-\int_{t_{1}}^{t_{2}} \delta W \mathrm{~d} t
$$

namely, the variation of the the action integral in correspondence of a possible motion is equal to the integral over time of minus the virtual work of the dissipative forces corresponding to the considered variation of the fields.

The way in which dissipation has to be introduced in saturated porous media models is still under debate, see for instance [21]. In particular according to the effectiveness of the hypothesis of separation of scales, between the local and macroscopic level, Darcy's or Stokes' effects are accounted for. We refer the interested reader to [10 for a complete discussion of this problem.

In this paper we shall consider the so-called Darcy effect, i.e., the dissipation due to forces proportional to the velocity of the fluid component measured with respect to the solid. By following phenomenological arguments [3] or by developing suitable homogenization schemes [14] it is seen that the fluid flow is, in this case, controlled by the permeability of the porous material. A natural expression for the virtual work of 
the dissipation forces acting on the fluid component and taking into account the Darcy effect is

$$
\delta W:=-\int_{B_{t}} D\left[v_{\mathrm{f}}(x, t)-v_{\mathrm{s}}(x, t)\right]\left[\delta \chi_{\mathrm{f}}\left(\chi_{\mathrm{f}}^{-1}(x, t), t\right)-\delta \chi_{\mathrm{s}}\left(\chi_{\mathrm{s}}^{-1}(x, t), t\right)\right] \mathrm{d} x
$$

where $D>0$ is a constant proportional to the inverse of permeability and $\delta \chi_{\mathrm{f}}$ is the variation of the field $\chi_{\mathrm{f}}$ induced by the independent variations $\delta \chi_{\mathrm{s}}$ and $\delta \phi$. The quantity $-D\left[v_{\mathrm{f}}-v_{\mathrm{s}}\right]$ is the dissipative force density (relative to the current configuration space volume) which depends on the kinematic fields only through the velocity of the fluid component relative to the solid. Apparently equation (2.5) for the virtual work of Darcy dissipative forces is consistent with the classical expression of Darcy dissipation associated with the viscous flow through a porous continuum, see [11].

Following the recipe described above we have to express the virtual work in terms of the field $\chi_{\mathrm{s}}$ and $\phi$. In [10] it has been proven that

$$
\delta W=-\int_{B_{\mathrm{s}}} R \delta \phi \mathrm{d} X_{\mathrm{s}} \quad \text { with } \quad R:=D \frac{1}{\left(\phi^{\prime}\right)^{2}} \dot{\phi}\left(\chi_{\mathrm{s}}^{\prime}\right)^{3}
$$

In order to write explicitly the variation of the action we specify, now, the form of the Lagrangian density. In the sequel we shall not consider the inertial effects, so that, the Lagrangian density will be the opposite of the potential energy density associated to both the internal and external conservative forces. As it has been shown in [22] (see equation (18) therein) it is reasonable to assume that the potential energy density depends on the space and time variable only via two physically relevant functions: the strain of the solid and a properly normalized fluid mass density [6 6, 22].

More precisely, consider the Jacobian $\chi_{\mathrm{s}}^{\prime}\left(X_{\mathrm{s}}, t\right)$ of the solid placement map, which measures the ratio between current and reference volumes of the solid component, and let

$$
\varepsilon\left(X_{\mathrm{s}}, t\right):=\left[\left(\chi_{\mathrm{s}}^{\prime}\left(X_{\mathrm{s}}, t\right)\right)^{2}-1\right] / 2
$$

be the strain field. Let $\varrho_{0, \mathrm{f}}: B_{\mathrm{f}} \rightarrow \mathbb{R}$ be the fluid reference density; we define the fluid mass density field

$$
m_{\mathrm{f}}\left(X_{\mathrm{s}}, t\right):=\varrho_{0, \mathrm{f}}\left(\phi\left(X_{\mathrm{s}}, t\right)\right) \phi^{\prime}\left(X_{\mathrm{s}}, t\right)
$$

Assuming that the mass is conserved, it is proven [22] that the field $m_{\mathrm{f}}$ can be interpreted as the fluid mass density measured with respect to the solid reference volume.

We assume the total potential energy density $\Phi$ to depend on the fields $m_{\mathrm{f}}$ and $\varepsilon$ and on their space derivative $m_{\mathrm{f}}^{\prime}$ and $\varepsilon^{\prime}$. Since $m_{\mathrm{f}}=\varrho_{0, \mathrm{f}}(\phi) \phi^{\prime}, m_{\mathrm{f}}^{\prime}=\varrho_{0, \mathrm{f}}^{\prime}(\phi)\left(\phi^{\prime}\right)^{2}+\varrho_{0, \mathrm{f}}(\phi) \phi^{\prime \prime}$, $\varepsilon=\left(\left(\chi_{\mathrm{s}}^{\prime}\right)^{2}-1\right) / 2$, and $\varepsilon^{\prime}=\chi_{\mathrm{s}}^{\prime} \chi_{\mathrm{s}}^{\prime \prime}$, we have that the Lagrangian density $\mathscr{L}=-\Phi$ depends on the space and time variables through the fields $\phi, \phi^{\prime}, \phi^{\prime \prime}, \chi_{\mathrm{s}}^{\prime}$, and $\chi_{\mathrm{s}}^{\prime \prime}$.

By a standard variational computation, see [10] for details, we finally get the equations of motion

$$
\left[\chi_{\mathrm{s}}^{\prime}\left(\frac{\partial \Phi}{\partial \varepsilon}-\left(\frac{\partial \Phi}{\partial \varepsilon^{\prime}}\right)^{\prime}\right)\right]^{\prime}=0 \text { and } \varrho_{0, \mathrm{f}}(\phi)\left[\frac{\partial \Phi}{\partial m_{\mathrm{f}}}-\left(\frac{\partial \Phi}{\partial m_{\mathrm{f}}^{\prime}}\right)^{\prime}\right]^{\prime}=R
$$

and the boundary conditions

$$
\begin{aligned}
\left\{\frac{\partial \Phi}{\partial m_{\mathrm{f}}^{\prime}} \varrho_{0, \mathrm{f}}(\phi) \delta \phi^{\prime}+\left[\left(\frac{\partial \Phi}{\partial m_{\mathrm{f}}}\right.\right.\right. & \left.\left.-\left(\frac{\partial \Phi}{\partial m_{\mathrm{f}}^{\prime}}\right)^{\prime}\right) \varrho_{0, \mathrm{f}}(\phi)+\frac{\partial \Phi}{\partial m_{\mathrm{f}}^{\prime}} \varrho_{0, \mathrm{f}}^{\prime}(\phi) \phi^{\prime}\right] \delta \phi \\
& \left.+\frac{\partial \Phi}{\partial \varepsilon^{\prime}} \chi_{\mathrm{s}}^{\prime} \delta \chi_{\mathrm{s}}^{\prime}+\left(\frac{\partial \Phi}{\partial \varepsilon}-\left(\frac{\partial \Phi}{\partial \varepsilon^{\prime}}\right)^{\prime}\right) \chi_{\mathrm{s}}^{\prime} \delta \chi_{\mathrm{s}}\right\}_{\ell_{1}}^{\ell_{2}}=0
\end{aligned}
$$


We remark that, though they are partially written in terms of the fields $m_{\mathrm{f}}$ and $\varepsilon$, the equations (2.9) are evolution equations for the two fields $\chi_{\mathrm{s}}$ and $\phi$. Only under a brute approximation, see the geometrical linearization discussed in Section 2.3, they will reduce to a set of evolutionary equations for the fields $m_{\mathrm{f}}$ and $\varepsilon$.

\subsection{Equations of motion under geometrical linearization}

In this section we rewrite the equations of motion of the poromechanical system under the so called geometrical linearization assumption, namely, in the case when only small elastic strains are present in the system. From now on we shall assume that $\varrho_{0, \mathrm{f}}$ is constant. We first introduce the displacement fields $u\left(X_{\mathrm{S}}, t\right)$ and $w\left(X_{\mathrm{S}}, t\right)$ by setting

$$
\chi_{\mathrm{s}}\left(X_{\mathrm{s}}, t\right)=X_{\mathrm{S}}+u\left(X_{\mathrm{s}}, t\right) \text { and } \phi\left(X_{\mathrm{s}}, t\right)=X_{\mathrm{s}}+w\left(X_{\mathrm{s}}, t\right)
$$

for any $X_{\mathrm{s}} \in B_{\mathrm{S}}$ and $t \in \mathbb{R}$. We then assume that $u$ and $w$ are small, together with their space and time derivatives, and write the equations of motion up to the first order in $u, w$, and derivatives. By using (2.7), (2.8), and recalling the definition of $R$ in (2.6), we get

$$
m_{\mathrm{f}}=\varrho_{0, \mathrm{f}}\left(1+w^{\prime}\right), m:=m_{\mathrm{f}}-\varrho_{0, \mathrm{f}}=\varrho_{0, \mathrm{f}} w^{\prime}, \varepsilon \approx u^{\prime}, \text { and } R \approx D \dot{w},
$$

where $\approx$ means that all the terms of order larger than one have been neglected.

We have introduced above the field $m$. In the following we shall imagine $\Phi$ as a function of $m$ and $m^{\prime}$ and the equations of motion and the boundary conditions will be written in terms of this field. We get the equations of motion

$$
\left[(1+\varepsilon)\left(\frac{\partial \Phi}{\partial \varepsilon}-\left(\frac{\partial \Phi}{\partial \varepsilon^{\prime}}\right)^{\prime}\right)\right]^{\prime}=0 \text { and } \varrho_{0, \mathrm{f}}\left[\frac{\partial \Phi}{\partial m}-\left(\frac{\partial \Phi}{\partial m^{\prime}}\right)^{\prime}\right]^{\prime}=D \dot{w}
$$

and the associated boundary conditions

$$
\left\{\frac{\partial \Phi}{\partial \varepsilon^{\prime}} \delta \varepsilon+\frac{\partial \Phi}{\partial m^{\prime}} \delta m+\left[\left(\frac{\partial \Phi}{\partial m}-\left(\frac{\partial \Phi}{\partial m^{\prime}}\right)^{\prime}\right) \varrho_{0, \mathrm{f}}\right] \delta w+\left(\frac{\partial \Phi}{\partial \varepsilon}-\left(\frac{\partial \Phi}{\partial \varepsilon^{\prime}}\right)^{\prime}\right) \delta u\right\}_{\ell_{1}}^{\ell_{2}}=0
$$

Recalling that in our approximation $m=\varrho_{0, \mathrm{f}} w^{\prime}$, see the second among equations (2.12), we have that the equations (2.13) are evolution equations for the fields $m$ and $\varepsilon$. Moreover, as remarked in [10], provided the potential energy density $\Phi$ depends on the space derivatives $m^{\prime}$ and $\varepsilon^{\prime}$ of the fields $m$ and $\varepsilon$ as a quadratic form with constant coefficients, it follows that the second between the equations of motion (2.13) becomes a Cahn-Hilliard-like equation for the field $m$ with driving field still depending parametrically on $\varepsilon$.

Note, also, that the equations 2.14 are an extension to the case of second gradient elasticity of classical natural and essential boundary conditions [13. By choosing in two different ways the boundary condition we shall obtain two different physically relevent boundary value problems.

\subsection{The zero chemical potential problem}

A set of boundary conditions implying that 2.14) are satisfied is

$$
\left(\frac{\partial \Phi}{\partial \varepsilon^{\prime}} \delta \varepsilon+\frac{\partial \Phi}{\partial m^{\prime}} \delta m\right)_{\ell_{1}, \ell_{2}}=\left[\frac{\partial \Phi}{\partial m}-\left(\frac{\partial \Phi}{\partial m^{\prime}}\right)^{\prime}\right]_{\ell_{1}, \ell_{2}}=\left[\frac{\partial \Phi}{\partial \varepsilon}-\left(\frac{\partial \Phi}{\partial \varepsilon^{\prime}}\right)^{\prime}\right]_{\ell_{1}, \ell_{2}}=0
$$


where the notation above means that the functions in brackets are evaluated both in $\ell_{1}$ and $\ell_{2}$. With this choice it is possible to fix the boundary conditions directly on fields $m$ and $\varepsilon$ (and derivatives).

The first equation (2.15) is the additional boundary condition due to the presence of the gradient terms in the potential energy density $\Phi$. This equation specifies essential boundary conditions on the derivatives of the displacement fields or natural boundary conditions on the so called double forces, see [15]. The generalized essential boundary conditions can be read as a prescription on the derivative of the independent fields $\chi_{\mathrm{s}}$ and $\phi$, see equations (2.7) and (2.8); whilst the extended natural boundary conditions prescribe, on one hand, the additional forces which the solid continuum is able to balance at the boundary and, on the other, the wetting properties of the fluid which fills the pores 23 .

The second and the third equations (2.15) provide natural boundary conditions prescribing the chemical potential of the fluid and the traction exerted on the overall porous solid, respectively. These conditions extend the classical ones (which can be easily found in the literature) for a porous solid suffering internal stresses due to applied tractions and for a saturating fluid with fixed chemical potential; see [2] for more details. In this case we use the words generalized traction and chemical potential because of the additional contribution to stress $(\partial \Phi / \partial \varepsilon)$ and chemical potential $(\partial \Phi / \partial m)$ provided by the spatial derivatives of the corresponding hyperstress fields, say $\left(\partial \Phi / \partial \varepsilon^{\prime}\right)^{\prime}$ and $\left(\partial \Phi / \partial m^{\prime}\right)^{\prime}$.

Finally, we note that, by exploiting the third boundary condition 2.15 in $\ell_{1}$, we get the PDE problem

$$
\left\{\begin{array}{l}
\frac{\partial \Phi}{\partial \varepsilon}-\left(\frac{\partial \Phi}{\partial \varepsilon^{\prime}}\right)^{\prime}=0 \text { and } \varrho_{0, \mathrm{f}}^{2}\left[\frac{\partial \Phi}{\partial m}-\left(\frac{\partial \Phi}{\partial m^{\prime}}\right)^{\prime}\right]^{\prime \prime}=D \dot{m} \\
\left(\frac{\partial \Phi}{\partial \varepsilon^{\prime}} \delta \varepsilon+\frac{\partial \Phi}{\partial m^{\prime}} \delta m\right)_{\ell_{1}, \ell_{2}}=\left[\frac{\partial \Phi}{\partial m}-\left(\frac{\partial \Phi}{\partial m^{\prime}}\right)^{\prime}\right]_{\ell_{1}, \ell_{2}}=0
\end{array}\right.
$$

that will be called the zero chemical potential problem.

\subsection{The one-side impermeable problem}

In the zero chemical potential problem we can imagine that the porous one-dimensional system is plunge into a fluid reservoir and that at the two boudaries the fluid can get in and out from the sample freely. A rather different very interesting situation in applications, see the discussion provided in the Introduction, is the one in which one of the two boudaries is not permeable to the fluid.

This physical situation can be mathematically implemented as follows. Consider the third term of boundary condition (2.14), which is zero when zero chemical potential is prescribed at the boundary, see equation (2.16). According with classical arguments of variational calculus it vanishes also when the variation of the fluid displacement, $\delta w$, vanishes, which corresponds to account for essential rather than natural boundary conditions. From the physical point of view, assuming $\delta w=0$ implies the fluid particle, occuping the same current place as the solid particle which remains at the boundary, to be always the same during evolution. In other words assuming that, at any time $t$, $\delta w$ equals zero, implies the seepage velocity, say $v=u_{\mathrm{f}}-u_{\mathrm{s}}=-\dot{w}$, to identically vanish and therefore the boundary to be impermeable to the fluid flow. Considering the bulk 
equation $(2.13)_{2}$ this boundary condition can therefore be rephrased as follows:

$$
v=-\frac{\varrho_{0, \mathrm{f}}}{D}\left(\frac{\partial \Phi}{\partial m}-\left(\frac{\partial \Phi}{\partial m^{\prime}}\right)^{\prime}\right)^{\prime}=0
$$

Thus, it follows that a set of boundary conditions implying that 2.14 are satisfied is

$$
\begin{aligned}
\left(\frac{\partial \Phi}{\partial \varepsilon^{\prime}} \delta \varepsilon+\frac{\partial \Phi}{\partial m^{\prime}} \delta m\right)_{\ell_{1}} & =\left[\left(\frac{\partial \Phi}{\partial m}-\left(\frac{\partial \Phi}{\partial m^{\prime}}\right)^{\prime}\right)^{\prime}\right]_{\ell_{2}} \\
& =\left[\frac{\partial \Phi}{\partial m}-\left(\frac{\partial \Phi}{\partial m^{\prime}}\right)^{\prime}\right]_{\ell_{1}}=\left[\frac{\partial \Phi}{\partial \varepsilon}-\left(\frac{\partial \Phi}{\partial \varepsilon^{\prime}}\right)^{\prime}\right]_{\ell_{1}, \ell_{2}}=0
\end{aligned}
$$

Finally, we note that, by exploiting the third boundary condition 2.15 in $\ell_{1}$, we get the PDE problem

$$
\left\{\begin{array}{l}
\frac{\partial \Phi}{\partial \varepsilon}-\left(\frac{\partial \Phi}{\partial \varepsilon^{\prime}}\right)^{\prime}=0 \text { and } \varrho_{0, \mathrm{f}}^{2}\left[\frac{\partial \Phi}{\partial m}-\left(\frac{\partial \Phi}{\partial m^{\prime}}\right)^{\prime}\right]^{\prime \prime}=D \dot{m} \\
\left(\frac{\partial \Phi}{\partial \varepsilon^{\prime}} \delta \varepsilon+\frac{\partial \Phi}{\partial m^{\prime}} \delta m\right)_{\ell_{1}, \ell_{2}}=\left[\left(\frac{\partial \Phi}{\partial m}-\left(\frac{\partial \Phi}{\partial m^{\prime}}\right)^{\prime}\right)^{\prime}\right]_{\ell_{2}}^{=}\left[\frac{\partial \Phi}{\partial m}-\left(\frac{\partial \Phi}{\partial m^{\prime}}\right)^{\prime}\right]_{\ell_{1}}=0
\end{array}\right.
$$

that will be called the one-side impermeable problem.

\subsection{The stationary problem}

In the above section we have introduced two different physically interesting problems for the porous medium we are studying by specifying two different sets of boundary conditions. The stationary solution of those two problems are the solution of the two differential equations

$$
\frac{\partial \Phi}{\partial \varepsilon}-\left(\frac{\partial \Phi}{\partial \varepsilon^{\prime}}\right)^{\prime}=0 \text { and }\left[\frac{\partial \Phi}{\partial m}-\left(\frac{\partial \Phi}{\partial m^{\prime}}\right)^{\prime}\right]^{\prime \prime}=0
$$

with the boundary conditions provided respectiveley in $(2.16)$ and $(2.19)$ for the zero chemical potential and the one-side impermeable problems.

It is easy to show that in both cases the boundary conditions imply that, see also 10 , equation (40) and related discussion], $\partial \Phi / \partial m-\left(\partial \Phi / \partial m^{\prime}\right)^{\prime}=0$, so that for both the problems the stationary solutions are the solutions of the PDE problem

$$
\left\{\begin{array}{l}
\frac{\partial \Phi}{\partial \varepsilon}-\left(\frac{\partial \Phi}{\partial \varepsilon^{\prime}}\right)^{\prime}=0 \text { and }\left[\frac{\partial \Phi}{\partial m}-\left(\frac{\partial \Phi}{\partial m^{\prime}}\right)^{\prime}\right]^{\prime \prime}=0 \\
\left(\frac{\partial \Phi}{\partial \varepsilon^{\prime}} \delta \varepsilon+\frac{\partial \Phi}{\partial m^{\prime}} \delta m\right)_{\ell_{1}, \ell_{2}}=0
\end{array}\right.
$$

\subsection{Dissipative character of the evolution}

Besides the stationary solutions, another common features of the zero chemical potential and the one-side impermeable problems is the dissipative character of the dynamics.

Indeed, the dissipative character of the physical problem we are studying reflects in the existence of the not increasing energy functional

$$
F\left(\chi_{\mathrm{s}}, \phi\right):=\int_{\ell_{1}}^{\ell_{2}} \Phi\left(m^{\prime}, \varepsilon^{\prime}, m, \varepsilon\right) \mathrm{d} X_{\mathrm{s}}
$$


for both the systems $(2.16)$ and $(2.19)$. To prove this we compute the time derivative of the functional $F$ evaluated on the fields $\chi_{\mathrm{s}}\left(X_{\mathrm{s}}, t\right)$ and $\phi\left(X_{\mathrm{s}}, t\right)$. We first get

$$
\frac{\mathrm{d} F}{\mathrm{~d} t}=\int_{\ell_{1}}^{\ell_{2}}\left[\frac{\partial \Phi}{\partial \varepsilon^{\prime}} \dot{\varepsilon}^{\prime}+\frac{\partial \Phi}{\partial m^{\prime}} \dot{m}^{\prime}+\frac{\partial \Phi}{\partial \varepsilon} \dot{\varepsilon}+\frac{\partial \Phi}{\partial m} \dot{m}\right] \mathrm{d} X_{\mathrm{s}}
$$

and, by integrating by parts, we obtain

$$
\frac{\mathrm{d} F}{\mathrm{~d} t}=\left[\frac{\partial \Phi}{\partial \varepsilon^{\prime}} \dot{\varepsilon}+\frac{\partial \Phi}{\partial m^{\prime}} \dot{m}\right]_{\ell_{1}}^{\ell_{2}}+\int_{\ell_{1}}^{\ell_{2}}\left[\left(\frac{\partial \Phi}{\partial m}-\left(\frac{\partial \Phi}{\partial m^{\prime}}\right)^{\prime}\right) \dot{m}+\left(\frac{\partial \Phi}{\partial \varepsilon}-\left(\frac{\partial \Phi}{\partial \varepsilon^{\prime}}\right)^{\prime}\right) \dot{\varepsilon}\right] \mathrm{d} X_{\mathrm{s}}
$$

Assume, now, that the fields $m$ and $\varepsilon$ are solution either the problem (2.16) or (2.19). Then,

$$
\frac{\mathrm{d} F}{\mathrm{~d} t}=\frac{\varrho_{0, \mathrm{f}}^{2}}{D} \int_{\ell_{1}}^{\ell_{2}}\left(\frac{\partial \Phi}{\partial m}-\left(\frac{\partial \Phi}{\partial m^{\prime}}\right)^{\prime}\right)\left(\frac{\partial \Phi}{\partial m}-\left(\frac{\partial \Phi}{\partial m^{\prime}}\right)^{\prime}\right)^{\prime \prime} \mathrm{d} X_{\mathrm{s}}
$$

Finally, by integrating by parts and by using the boundary conditions, in both cases we find

$$
\frac{\mathrm{d} F}{\mathrm{~d} t}=-\frac{\varrho_{0, \mathrm{f}}^{2}}{D} \int_{\ell_{1}}^{\ell_{2}}\left[\left(\frac{\partial \Phi}{\partial m}-\left(\frac{\partial \Phi}{\partial m^{\prime}}\right)^{\prime}\right)^{\prime}\right]^{2} \mathrm{~d} X_{\mathrm{s}}
$$

which proves that the functional $F$ in not increasing along the motions of the CahnHilliard-like systems (2.16) and 2.19).

\subsection{Summary of the section}

In conclusion, in this section, we have written the equations of motions (2.13) and the associated boundary conditions (2.14) under the geometrical linearization assumption. We have then introduced suitable boundary conditions to model mathematically the zero chemical potential and one-side impermeability problems. We have thus obtained the two PDE problems (2.16) and (2.19). Moreover, we have remarked that the stationary solutions of those problems are given by the same system of equations (2.21). Finally, we have proven that the energy functional $(2.22)$ does not increase along the solutions of both the two systems (2.16) and (2.19), which suggests that in both cases the motions will tend asymptotically to the stationary profiles.

\section{A special model}

We apply, now, the theory developed above to the special model introduced in $[6] 8$ and whose stationary behavior has been widely discussed in those papers.

We first specialize the model we are studying by choosing the second gradient part of the dimensionless potential energy, that is we assume

$$
\Phi\left(m^{\prime}, \varepsilon^{\prime}, m, \varepsilon\right):=\frac{1}{2}\left[k_{1}\left(\varepsilon^{\prime}\right)^{2}+2 k_{2} \varepsilon^{\prime} m^{\prime}+k_{3}\left(m^{\prime}\right)^{2}\right]+\Psi(m, \varepsilon)
$$

with $k_{1}, k_{3}>0, k_{2} \in \mathbb{R}$ such that $k_{1} k_{3}-k_{2}^{2} \geq 0$. These parameters provide energy penalties for the formation of interfaces; they have the physical dimensions of squared lengths and, according with the above mentioned conditions, provide a well-grounded 
identification of the intrinsic characteristic lengths of the one-dimensional porous continuum.

Moreover, we consider the following expression for the total potential energy density in the perspective of describing the transition between a fluid-poor and a fluid-rich phase

$$
\Psi(m, \varepsilon):=\frac{\alpha}{12} m^{2}\left(3 m^{2}-8 b \varepsilon m+6 b^{2} \varepsilon^{2}\right)+\Psi_{\mathrm{B}}(m, \varepsilon)
$$

where

$$
\Psi_{\mathrm{B}}(m, \varepsilon):=p \varepsilon+\frac{1}{2} \varepsilon^{2}+\frac{1}{2} a(m-b \varepsilon)^{2}
$$

is the Biot potential energy density [4], $a>0$ is the ratio between the fluid and the solid rigidity, $b>0$ is a coupling between the fluid and the solid component, $p>0$ is the external pressure, and $\alpha>0$ is a material parameter responsible for the showing up of an additional equilibrium.

In the papers $[6-8]$ we have studied the stationary problem $(2.21)$ corresponding to the potential energies (3.24) and (3.25). In this section we recall briefly those results and extend the paremetrical study in $[10]$ with respect to the second gradient coefficients $k_{1}, k_{2}$, and $k_{3}$.

First of all we write explicitly the stationary problem corresponding to the potential energies (3.24) and (3.25). By 2.21) we get

$$
\left\{\begin{array}{l}
-(2 / 3) \alpha b m^{3}+\alpha b^{2} m^{2} \varepsilon+p+\varepsilon-a b(m-b \varepsilon)-k_{1} \varepsilon^{\prime \prime}-k_{2} m^{\prime \prime}=0 \\
\alpha m^{3}-2 \alpha b m^{2} \varepsilon+\alpha b^{2} m \varepsilon^{2}+a(m-b \varepsilon)-k_{2} \varepsilon^{\prime \prime}-k_{3} m^{\prime \prime}=0 \\
\left(\left(k_{1} \varepsilon^{\prime}+k_{2} m^{\prime}\right) \delta \varepsilon+\left(k_{2} \varepsilon^{\prime}+k_{3} m^{\prime}\right) \delta m\right)_{\ell_{1}, \ell_{2}}=0
\end{array}\right.
$$

where the last line is the boundary condition.

\subsection{Phases: constant stationary solutions}

In 6, 8, we have studied the constant solutions of (3.27) which are called phases of the system. We have proven that there exists a pressure $p_{\mathrm{c}}$, called critical pressure, such that for any $p \in\left[0, p_{\mathrm{c}}\right)$ there exists a single phase $\left(m_{\mathrm{S}}(p), \varepsilon_{\mathrm{S}}(p)\right)$, called the standard phase, which is very similar to the usual solution of the Biot model. For $p>p_{\mathrm{c}}$ a second phase $\left(m_{\mathrm{f}}(p), \varepsilon_{\mathrm{f}}(p)\right)$, richer in fluid with respect to the standard phase and hence called fluid-rich phase, appears. We underline that from now on $m_{\mathrm{f}}(p)$ indicates the increment of the fluid mass density $m$ evaluated in the fluid-rich phase of the porous system, parametrized by the consolidating pressure.

We have shown that the standard phase $\left(m_{\mathrm{s}}(p), \varepsilon_{\mathrm{S}}(p)\right)$ is the solution of the two equations $m=b \varepsilon$ and $p=f_{1}(\varepsilon)$, for any $p>0$, where $f_{1}(\varepsilon):=-\varepsilon-\alpha b^{4} \varepsilon^{3} / 3$. On the other hand the fluid-rich phase $\left(m_{\mathrm{f}}(p), \varepsilon_{\mathrm{f}}(p)\right)$ is the solution, with the smallest value of $\varepsilon$ (recall $\varepsilon \in(-1 / 2,0)$, so that the smallest value has indeed largest modulus), of the two equations $m=m_{+}(\varepsilon)$ and $p=f_{+}(\varepsilon)$, where

$m_{+}(\varepsilon)=\frac{b}{2}\left[\varepsilon+\sqrt{\varepsilon^{2}-\frac{4 a}{\alpha b^{2}}}\right] \quad$ and $\quad f_{+}(\varepsilon):=-\varepsilon+a b\left[m_{+}(\varepsilon)-b \varepsilon\right]-\alpha b^{2} \varepsilon m_{+}^{2}(\varepsilon)+\frac{2}{3} \alpha b m_{+}^{3}(\varepsilon)$

For $\varepsilon \leq-2 /(b \sqrt{\alpha / a})$ the function $f_{+}(\varepsilon)$ is positive, diverging to $+\infty$ for $\varepsilon \rightarrow-\infty$, and has a minimum at $\varepsilon_{\mathrm{c}}$ such that $f_{+}\left(\varepsilon_{\mathrm{c}}\right)=p_{\mathrm{c}}$; this explains why the fluid-rich phase is seen only for $p>p_{\mathrm{c}}$. 
Moreover it has been shown that for any $p>0$ the point $\left(m_{\mathrm{S}}(p), \varepsilon_{\mathbf{S}}(p)\right)$ is a minimum of the two variable potential energy $\Psi(m, \varepsilon)$ with $p$ fixed, while $\left(m_{\mathrm{f}}(p), \varepsilon_{\mathrm{f}}(p)\right)$ is a minimum for $p>p_{\mathrm{c}}$ and a saddle point for $p=p_{\mathrm{c}}$. For more details we refer to [8].

\subsection{Profiles: not constant stationary solutions}

In [7] it has been proven that there exists a unique value $p_{\mathrm{co}}$ of the pressure, called coexistence pressure, such that the potential energy of the two phases is equal. More precisely, it has been proven that the equation $\Psi\left(m_{\mathrm{s}}(p), \varepsilon_{\mathrm{s}}(p)\right)=\Psi\left(m_{\mathrm{f}}(p), \varepsilon_{\mathrm{f}}(p)\right)$ has the single solution $p_{\mathrm{co}}$.

The behavior of the system at the coexistence pressure is particularly interesting; from now on we shall always consider $p=p_{\text {co }}$ and, for this reason, we shall drop $p$ from the notation. When the external pressure is equal to $p_{\text {co }}$, none of the two above phases is favored and we ask if profiles connecting one phase to the other exist. More precisely, in [7] we have addressed the problem of the existence of a connection between the two phases, that is, a solution of the stationary problem (3.27) on $\mathbb{R}$ tending to the standard phase for $X_{\mathrm{s}} \rightarrow-\infty$ and to the fluid-rich one for $X_{\mathrm{s}} \rightarrow+\infty$. Using results in [1] we have proven that such a connection does exist provided $k_{1} k_{3}-k_{2}^{2}>0$.

We have also shown that, for $k_{1} k_{3}-k_{2}^{2}=0$ (degenerate case), the problem of finding a solution of the stationary problem can be reduced to the computation of a definite integral via a rephrasing of the problem as a one dimensional conservative mechanical system. We do not repeat here the discussion, but we refer the interested reader to [10, Section 4.2] and references therein.

In this section we just want to check that the behavior of the system out of the degenerate case does not change too much with respect to the degenerate case itself. In the not degenerate case, i.e., $k_{1} k_{3}-k_{2}^{2}>0$, in 7 we could prove the existence of a connection, but we were not able to find it explicitly. Studying in more detail the behavior of the solutions of the system (3.27) is a difficult task since, in this case, the problem cannot be rewritten as a one dimensional conservative mechanical system. We shall then study the problem numerically and solve the system (3.27) by means of the finite difference method powered by the Newton-Rapson algorithm. The substitution rule we use are standard, see, for instance [10, Appendix B].

Our results are depicted in the figure 3.1. Morally, in the three pictures, we first consider the case $k_{1}=k_{2}=k_{3}=10^{-3}$ and then vary one of the three parameters at time. Our results show that the general behavior of the connections does not change very much and that only minor details change.

\section{The not stationary problem}

In this section we discuss the not stationary solution of the problems 2.16) and 2.19. As stated in the Introduction our aim is that of finding out the effect of the impermeable boundary to the evolution fo the profiles.

The equations of motion describing the evolution of the system in the two cases read

$$
\left\{\begin{array}{l}
-(2 / 3) \alpha b m^{3}+\alpha b^{2} m^{2} \varepsilon+p+\varepsilon-a b(m-b \varepsilon)-k_{1} \varepsilon^{\prime \prime}-k_{2} m^{\prime \prime}=0 \\
\varrho_{0, \mathrm{f}}^{2}\left(\alpha m^{3}-2 \alpha b m^{2} \varepsilon+\alpha b^{2} m \varepsilon^{2}+a(m-b \varepsilon)-k_{2} \varepsilon^{\prime \prime}-k_{3} m^{\prime \prime}\right)^{\prime \prime}=D \dot{m}
\end{array}\right.
$$



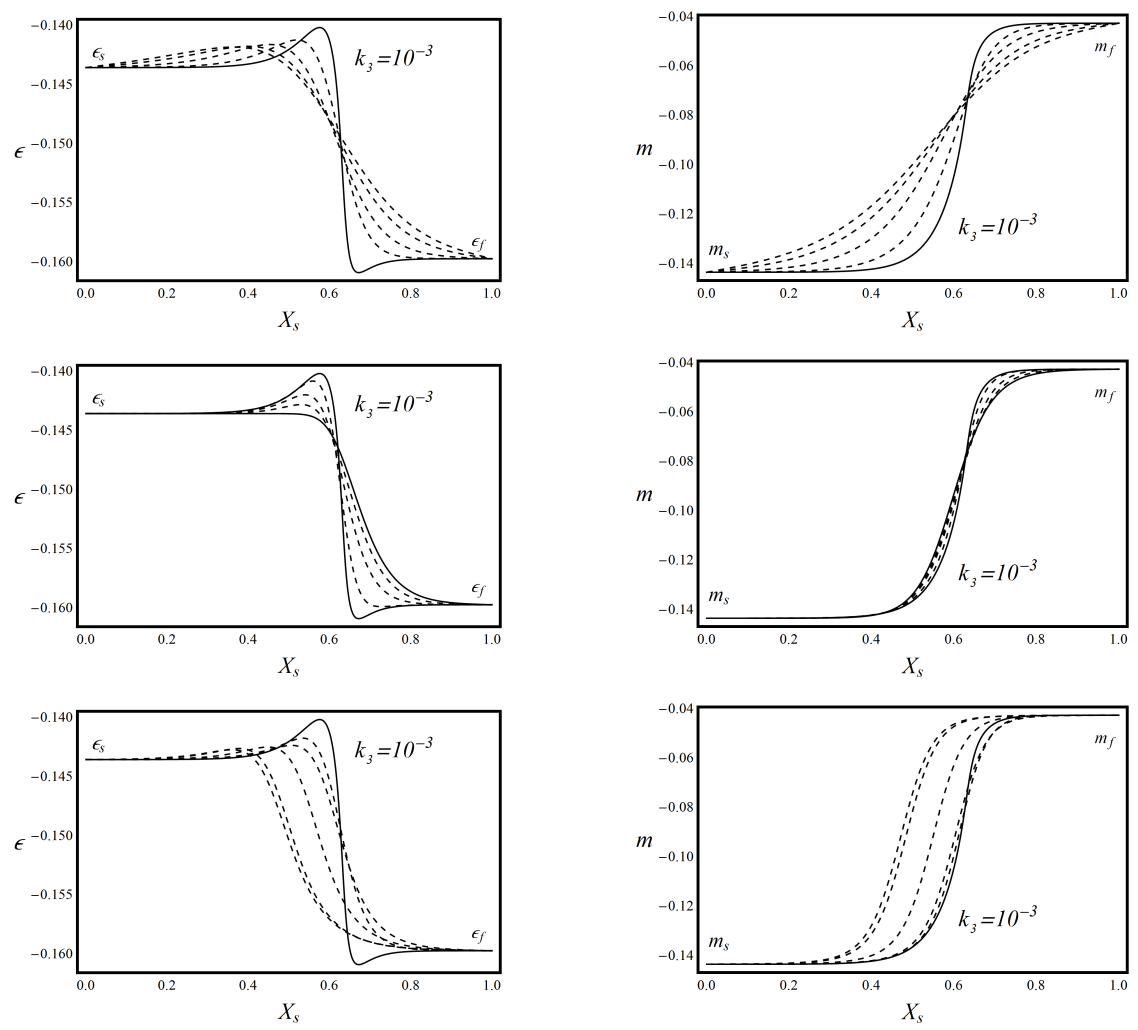

Figure 3.1: Soutions $\left(\varepsilon\left(X_{s}\right)\right.$ on the left and $m\left(X_{s}\right)$ on the right) of the stationary problem (3.27) with the Dirichlet boundary conditions $m(0)=m_{s}, \varepsilon(0)=\varepsilon_{s}, m(1)=$ $m_{f}$, and $\varepsilon(1)=\varepsilon_{f}$ on the finite interval [0,1], at the coexistence pressure for $a=0.5, b=$ 1 , and $\alpha=100$. The second gradient parameters are as follows. Bottom pictures: $k_{2}=k_{3}=10^{-3}, k_{1}=10^{-3}$ (solid line), and $k_{1}=10^{-2}, 0.4 \times 10^{-2}, 0.7 \times 10^{-2}, 0.8 \times$ $10^{-2}, 0.9 \times 10^{-2}$ (dotted lines). Middle pictures: $k_{1}=k_{3}=10^{-3}, k_{2}= \pm 10^{-3}$ (solid lines), and $k_{2}=-0.4 \times 10^{-} 3,0.2 \times 10^{-} 3,0.8 \times 10^{-} 3$ (dotted lines). Top pictures: $k_{1}=$ $k_{2}=10^{-3}, k_{3}=10^{-3}$ (solid line), and $k_{3}=10^{-2}, 0.2 \times 10^{-2}, 0.4 \times 10^{-2}, 0.7 \times 10^{-2}, 10^{-2}$ (dotted lines).

with the boundary conditions

$$
\left\{\begin{array}{l}
\left(\left(k_{2} m^{\prime}+k_{1} \varepsilon^{\prime}\right) \delta \varepsilon+\left(k_{3} m^{\prime}+k_{2} \varepsilon^{\prime}\right) \delta m\right)_{\ell_{1}, \ell_{2}}=0 \\
\left(\alpha m^{3}-2 \alpha b m^{2} \varepsilon+\alpha b^{2} m \varepsilon^{2}+a(m-b \varepsilon)-k_{2} \varepsilon^{\prime \prime}-k_{3} m^{\prime \prime}\right)_{\ell_{1}, \ell_{2}}=0
\end{array}\right.
$$

for the zero chemical potential problem 2.16 and

$$
\left\{\begin{array}{l}
\left(\left(k_{2} m^{\prime}+k_{1} \varepsilon^{\prime}\right) \delta \varepsilon+\left(k_{3} m^{\prime}+k_{2} \varepsilon^{\prime}\right) \delta m\right)_{\ell_{1}, \ell_{2}}=0 \\
\left(3 \alpha m^{2} m^{\prime}-4 \alpha b m m^{\prime} \varepsilon-2 \alpha b m^{2} \varepsilon^{\prime}+\alpha b^{2} m^{\prime} \varepsilon^{2}\right. \\
\left.\quad+2 \alpha b^{2} m \varepsilon \varepsilon^{\prime}+a\left(m^{\prime}-b \varepsilon^{\prime}\right)-k_{2} \varepsilon^{\prime \prime \prime}-k_{3} m^{\prime \prime \prime}\right)_{\ell_{2}}=0 \\
\left(\alpha m^{3}-2 \alpha b m^{2} \varepsilon+\alpha b^{2} m \varepsilon^{2}+a(m-b \varepsilon)-k_{2} \varepsilon^{\prime \prime}-k_{3} m^{\prime \prime}\right)_{\ell_{1}}=0
\end{array}\right.
$$


for the one-side impermeable problem 2.19.

We have studied the above PDE problem with the same numerical approach used for the stationary problem.

The dissipative evolution of the elastic strain $\varepsilon$ and the fluid mass density variations $m$, relative to the zero chemical potential boundary condition and the one-side impermeability condition, are discussed for different initial conditions, say: $(i)$ linear initial condition connecting the two phases, (ii) fluid-poor phase initial condition, (iii) fluid-rich phase intial condition. We remind that in the case of one side impermeability condition the chemical potential is still required to vanish at $X_{\mathrm{s}}=0$. In figures 4.24 .7 the corresponding profiles of the elastic strain, the fluid mass density variation and the seepage velocity (defined as in (2.17)) are reported at different times.

\subsection{Linear intial condition}

Figures 4.2 and 4.3 refer to the evolution of the system starting from spatially linear initial conditions for $\varepsilon$ and $m$ connecting the standard (fluid-poor phase) on the left to the fluid-rich phase on the right.

The evolution can be divided into two macroscopically different regimes: the first one is the formation of the connection profile, while the second one is a sort of travelling wave which represents the slow propagation of the profile towards the stationary state. The transition between the two above-mentioned regimes is associated to the showing up of a characteristic velocity profile (see, for instance, the three bottom rows in figure 4.2 that we shall call the late time velocity profile. In the following we will analyze separately the two regimes of the evolution, comparing the profiles relative to the considered boundary conditions.

Comparing figures 4.2 and 4.3 , one can observe that close to the boundary where impermeability is prescribed, strain and fluid density variations suffer a jump. This is mainly due to the delay of the system in modifying its initial state according with the prescribed boundary condition and, in particular, to the fact that no flow through the right boundary is allowed. On the other hand, when considering the zero chemical potential boundary condition, the formation of the profiles do not pass through this intermediate step. Consequently the second regime of the evolution process is reached more quickly and this is witnessed also by the formation of the characteristic late time velocity profile. In both cases the formation of the connection profile is accompanied by a significant swelling of the solid matrix in that part of the domain where a significant fluid admission is observed.

In order to understand the formation of the connection profile, i.e., the first part of the evolution, one has to look at the first four rows, corresponding to times $t=$ $0.05,0.2,0.3,0.75$, in figures 4.2 and 4.3 . Close to the left boundary the behavior of the zero chemical potential and that of the one-side impermeable problem are very similar. On the other hand, they are pretty different (as expected) close to the right boundary. Indeed, in both cases a fluid emission occurs through the left boundary point, as it can be deduced by noting that in the neighbourhood of the left boundary, the seepage velocity is negative (see the corresponding third column graphs).

In the zero chemical potential case the profile formation is due to two counterbalancing effects: fluid displacement from the central region of the sample, where a fluid excess (with respect to the stazionary state) is present at the initial times, towards the 
right and fluid supply through the right boundary point. This can be easily deduced by looking at the velocity profile which is positive in the central region and negative close to the right boundary (see the first three graphs from the top of the right column in figure 4.2. . On the other hand, in the one-side impermeable case, no fluid supply is possible through the right boundary point (notice that the velocity profile is zero in this case, see figure 4.3), so that the profile formation is due to the only effect of the central fluid displacement. As a result, in this last case the profile formation is slower and the profile is formed more to the right with respect to the zero chemical potential case. Notice, for instance, that the velocity peak at time $t=0.75$ is at 0.534 in the zero chemical potential case and at 0.574 in the one-side impermeable one.

At the end of the formation regime, the velocity profile assumes, in both case the peculiar late time shape with two maxima and to minima and intensity values significantly lower with respect to the previous time steps. This can be observed, for example in the zero chemical potential case, by comparing the value of the velocity peak a time $t=0.05$, where its intensity is about $5 \times 10^{-2}$, and time $t=0.75$ where its intensity is about $1.4 \times 10^{-4}$.

As we have already noticed, we remark that the profile formation is faster in the zero chemical potential boundary conditions than in the other case; indeed, the late time velocity profile is neatly formed at time $t=0.75$ in the first case, while in the second one it is necessary to wait till time $t=4$.

Once the characteristic late time velocity profile is formed, the slow propagation towards the stationary state starts. This motion, in this case of linear initial condition and, as we will see, in the case of the fluid-rich phase initial condition, is characterized by a negative peak intensity larger than that of the positive one. This property is due to the choice of the initial condition: indeed, since the profile motion towards stationarity proceeds from the left to the right, an amount of water has to go out from the medium, and this is reflected in a bigger negative part in the velocity profile.

Finally, we note that the stationary profile is reached in the one-side impermeable case faster than in the zero chemical potential one. It is important to remark that this is not a physically relevant effect, but is simply due to the choice of the initial condition. Indeed, due to our choice, in the one-side impermeable case the profile forms very close to the stationary one.

\subsection{Fluid-poor initial condition}

We discuss now the solution of the problems 4.29 e 4.30 with the constant fluidpoor profile as initial condition. Results are plotted in figures 4.4 and 4.5 as before, the three columns refer to $\varepsilon, m$, and the seepage velocity $v$, respectively.

As in the case of the linear initial condition and, as we will see, in the case of the fluid-rich phase initial condition, the evolution can be divided into two macroscopically different regimes: formation of the connection profile and slow travelling wave regime. Thus, the same physical arguments discussed in the previous case continue to be valid even in this case.

In the very early times of the profile formation, both for the zero chemical potential and the one-side impermeable cases, the evolution is characterized by a big deviation in the $\varepsilon$ profile, from the standard initial value, to large values outside the range $\left(\varepsilon_{f}, \varepsilon_{m}\right)$. A similar abrupt behavior is not observed for the fluid density profile: the deviation 
below the $m_{s}$ value (dashed lines), is present, but much smaller. The corresponding velocity profiles are characterized, in the neighbourhood of the right boundary, by large negative values in the zero chemical potential case and large positive values in the other one. This behavior has a rather clear physical interpretation: as a large amount of fluid has to be accumulated close to the right boundary, in the zero chemical potential case it is retrieved from the exterior through the right boundary point, while in the other case, due to the impermeability of the right boundary, such a fluid has to quickly arrive from the central part of the interior of the sample. As in the case of linear initial conditions this fluid accumulation implies a swelling of the solid skeleton, whose amplitude is much more significant with respect to that stemming from linear initial conditions.

It is very interesting to remark that when the density profile is almost formed, see the fifth row in figure 4.5 corresponding to time $t=11.1$, the seepage velocity attains negative values also close to the right boundary. We can intepret this fact by imagining that a certain amount of fluid is now present in that physical region and it starts to move to the right in order to push the profile towards the stationary position.

It is again possible to observe that, as in the previous case and for the same reason, a sort of discontinuity appears in the $\varepsilon$ profile in the one-side impermeable case (see figure 4.5 at time $t=0.7930105$ ).

Finally, once the profile is formed, again we observe the peculiar late time velocity graph. In this case, in contrast to the previous one, such a profile has a positive part bigger that the negative one. This is due to the fact that, for the present initial condition, it happens that the travelling wave has to propagate in the opposite direction.

\subsection{Fluid-rich inital condition}

We discuss now the solution of the problems 4.29 e 4.30 with the constant fluidrich profile as initial condition. Results are plotted in figures 4.6 and 4.7 as before, the three columns refer to $\varepsilon, m$, and the velocity, respectively.

In this case the system is started with an eccess of fluid throughout the sample. The profile matches the Dirichelet boundary condition on the right, so that the only phenomenon that has to happen is the fluid escape through the left boundary point. For this reason the evolutions in the zero chemical potential and the one-side impermeable one are very similar.

With respect to the cases discussed before it is interesting to remark that no sort of discontinuity appears in the $\varepsilon$ and $m$ profiles close to the right boundary. This is probably due to the fact that nothing relevant is happening close to such a boundary point.

The two evolutions show differences at times $t=0.8$ and $t=8$, see figures 4.6 and 4.7. Indeed, the velocity profiles are very similar at early times the positive part is almost the same in both cases, in the late steps the velocity profile concerning the evolution with the zero chemical potential has positive values in the middle of the porous medium and in the neighbourhood of the right boundary, unlike the velocity profile related to the evolution with the impermeability condition, where the velocity profile is all negative in the same region.

Once the profile is formed again the late time velocity graph shows up and slowly proceeds towards the stationary state moving from the left to the right. This is consistent with negative peak of the velocity which is greater than the positive one. Finally 
we can observe that a similar behavior as that relative to the fluid-poor initial condition can be detected concerning the presence of a kind of jump of the $\varepsilon$ profile outside the range $\left(\varepsilon_{f}, \varepsilon_{m}\right)$, which now concentrates close to the left boundary, while $m$ has a correspondent overshoot above the $m_{f}$ value. In this case however the jump amplitude is smaller than that of the previous one.

\section{Conclusions}

The occurrence of skeleton heterogeneous elastic strains and fluid density variations for a consolidating porous material have been modeled introducing a total potential energy capable for describing the transition between a fluid-poor and a fluid-rich phase inside the porous network. Owing to previous results $[7,10$ an extended description of the consolidation process has been implemented, within the framework of second gradient elasticity, which generalizes that provided by classical Terzaghi's equation. The capability of the model to accout for phase coexistence allows to capture the formation of heterogeneous equilibria quite similar to those arising during globally undrained tests when, at high level of confinement, the fluid can remain trapped into bands of localized strain.

Further development will be devoted to develop a parametric analysis of the consolidation process, with the aim of identifying the ranges of the applied pressure at which more general heterogeneous equilibria can be attained by the system.

\section{References}

[1] N. D. Alikakos and G. Fusco. On the connection problem for potentials with several global minima. Indiana University Mathematics Journal, 57(4):1871-1906, 2008.

[2] S. Baek and A. R. Srinivasa. Diffusion of a fluid through an elastic solid undergoing large elastic strain. International Journal of Non-Linear Mechanics, 39(2):201-218, 2004 .

[3] J. Bear. Dynamics of Fluids in Porous Media, Elsevier 1972.

[4] M. A. Biot. General theory of three-dimensional consolidation. Journal of Applied Physics, 12(2):155-164, 1941.

[5] P. Blanchard and E. Brüning. Variational Methods in Mathematical Physics, Springe 1992.

[6] E. N. M. Cirillo, N. Ianiro, and G. Sciarra. Solid-fluid segregation in saturated porous media. Poromechanics IV: Proceedings of the Fourth BIOT Conference on Poromechanics, 2009.

[7] E. N. M. Cirillo, N. Ianiro, and G. Sciarra. Phase coexistence in consolidating porous media. Physical Review E - Statistical, Nonlinear, and Soft Matter Physics, 81(6), 2010. 
[8] E. N. M. Cirillo, N. Ianiro, and G. Sciarra. Phase transition in saturated porous media: Pore-fluid segregation in consolidation. Physica D: Nonlinear Phenomena, 240(17):1345-1351, 2011.

[9] E. N. M. Cirillo, N. Ianiro, and G. Sciarra. Kink localization under asymmetric double-well potential. Physical Review E - Statistical, Nonlinear, and Soft Matter Physics, 86(4), 2012.

[10] E. N. M. Cirillo, N. Ianiro, and G. Sciarra. Allen-cahn and cahn-hilliard-like equations for dissipative dynamics of saturated porous media. Journal of the Mechanics and Physics of Solids, 61:629-651, 2013.

[11] O. Coussy. Poromechanics, Wiley 2004.

[12] C. W. Cryer. A comparison of the three-dimensional consolidation theories of biot and terzaghi. Quarterly Journal of Mechanics and Applied Mathematics, 16(4):401-412, 1963.

[13] F. Dell'Isola, G. Sciarra, and S. Vidoli. Generalized hooke's law for isotropic second gradient materials. Proceedings of the Royal Society A: Mathematical, Physical and Engineering Sciences, 465(2107):2177-2196, 2009.

[14] H. I. Ene and E. Sanchez-Palencia. Equations et phénomènes de surface pour l'ècoulement dans un modèle de milieux poreux $J$. de Mécanique, 14(1):73-108, 1975 .

[15] P. Germain. La méthode des puissances virtuelles en mécanique des milieux continus. I. Théorie du second gradient. J. de Mécanique, 12(2):235-274, 1973.

[16] D. Kolymbas. Compaction waves as phase transitions. Acta Mechanica, 107(14):171-181, 1994.

[17] J. Mandel. Consolidation des sols (étude mathématique). Geotechnique, 3(7):287299, 1953.

[18] M. Mokni and J. Desrues. Strain localization measurements in undrained planestrain biaxial tests on Hostun RF sand. Mech. Cohes.-Frict. Mater., 4:419-441, 1998.

[19] M. A. Mooney, G. Viggiani, and R.J. Finno. Undrained shear band elastic strain in granular media. Journal of engineering mechanics, 123:577-585, 1997.

[20] R. J. Nichols, R. S. J. Sparks, and C. J. N. Wilson. Experimental studies of the fluidization of layered sediments and the formation of fluid escape structures. Sedimentology, 41(2):233-253, 1994.

[21] D. A. Nield. The modeling of viscous dissipation in a saturated porous medium. Journal of Heat Transfer, 129(10):1459-1463, 2007.

[22] G. Sciarra, F. Dell-Isola, N. Ianiro, and A. Madeo. A variational deduction of second gradient poroelasticity part I: General theory. Journal of Mechanics of Materials and Structures, 3(3):507-526, 2008. 
[23] P. Seppecher. Etude des conditions aux limites en théorie du second gradient: Cas de la capillarité. C.R. Acad. Sci. Paris, 309(6):497-502, 1989.

[24] J. Sulem and H. Ouffroukh. Shear banding in drained and undrained triaxial tests on a saturated sandstone: Porosity and permeability evolution. International journal of rock mechanics and mining sciences, 43:292-502, 2006.

[25] I. Vardoulakis. Fluidisation in artesian flow conditions: Hydrochemically stable granular media. Geotechnique, 54(2):117-130, 2004.

[26] I. Vardoulakis. Fluidisation in artesian flow conditions: Hydromechanically unstable granular media. Geotechnique, 54(3):165-177, 2004. 

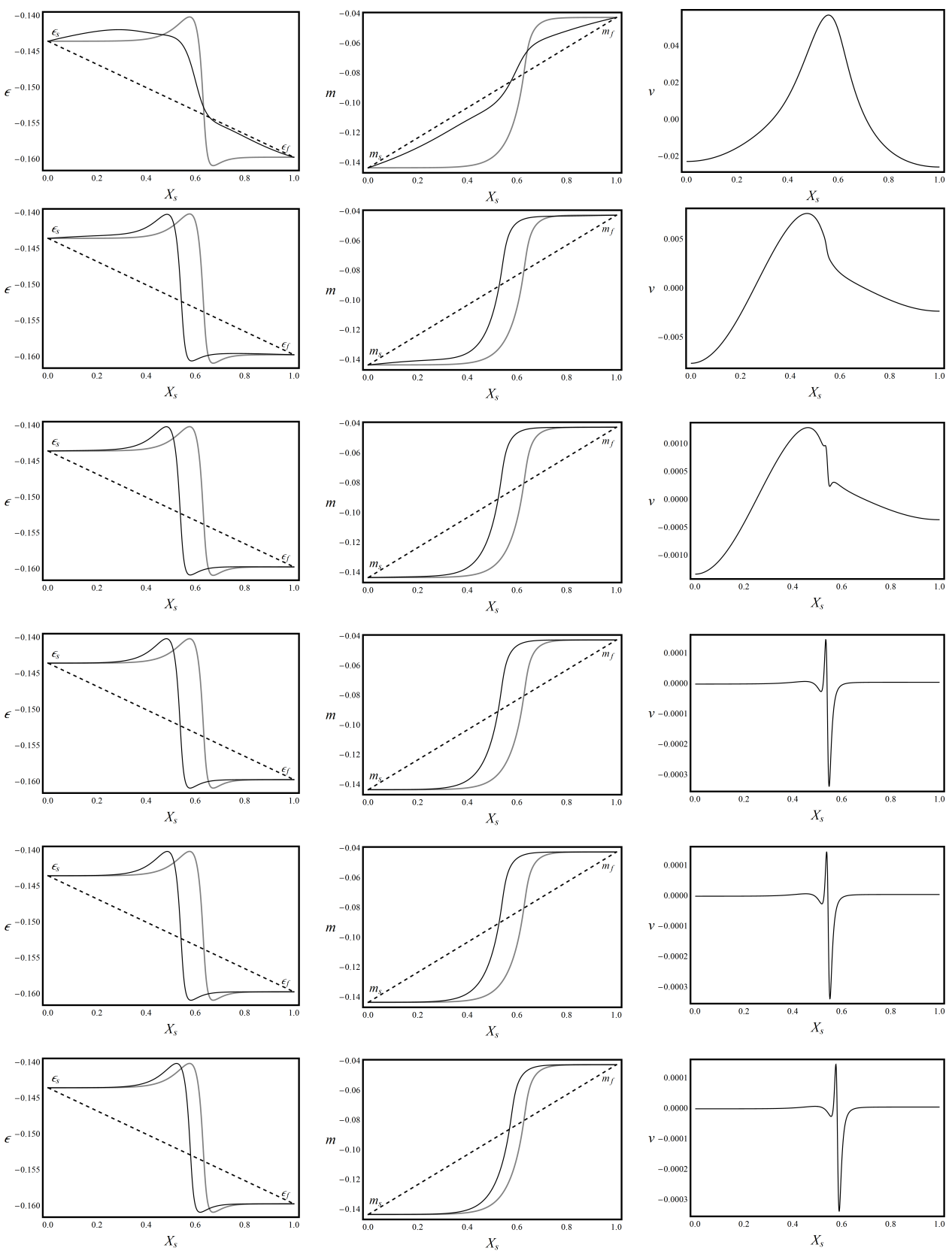

Figure 4.2: Profiles (black lines) $\varepsilon\left(X_{s}, t\right), m\left(X_{s}\right)$ and $v\left(X_{s}\right)$ for the zero chemical potential problem obtained by solving the non-stationary system with a linear initial state (dashed lines). We used Dirichelet boundary conditions $m(0)=m_{s}, \varepsilon(0)=$ $\varepsilon_{s}, m(1)=m_{f}, \varepsilon(1)=\varepsilon_{f}$ on the finite interval $[0,1]$, at the coexistence pressure for $a=0.5, b=1, \alpha=100, k_{1}=k_{2}=k_{3}=10^{-3}$. Profiles at times $t=0.05, t=0.2, t=0.3, t=0.75, t=4, t=40$ in lexicographic order. The gray lines represent stationary profiles. 

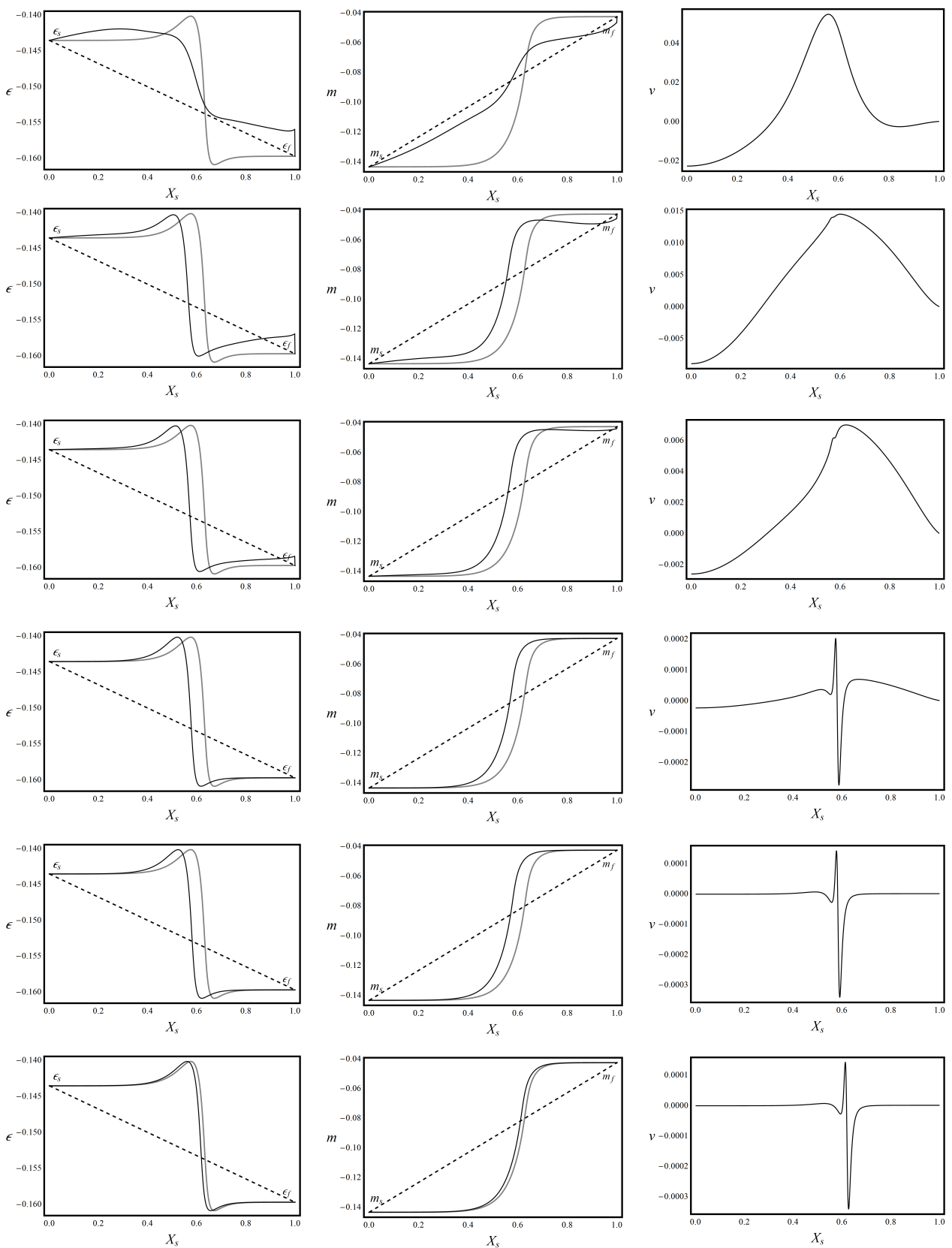

Figure 4.3: The same as in figure 4.2 but with the one side impermeability condition. 

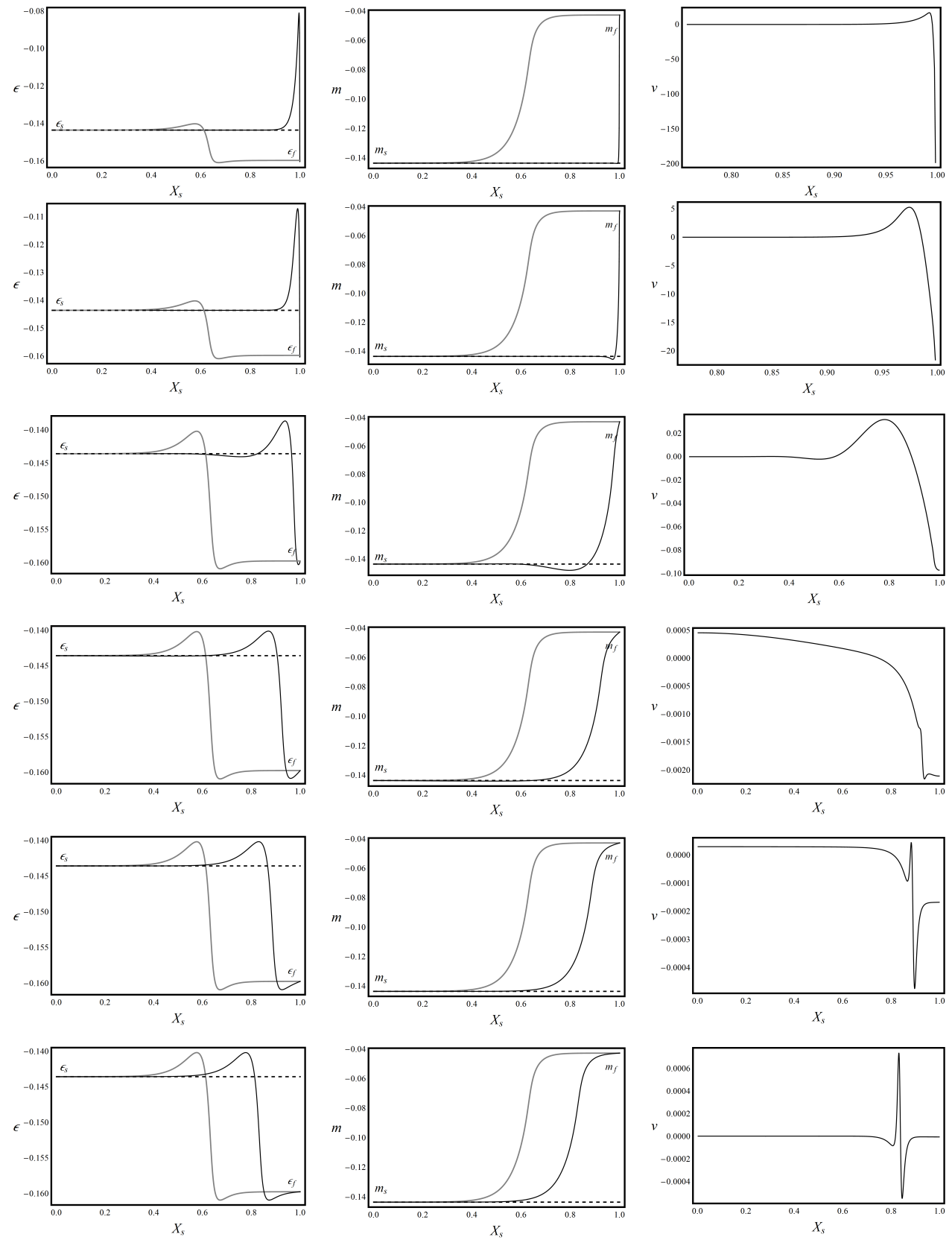

Figure 4.4: Profiles (black lines) $\varepsilon\left(X_{s}, t\right), m\left(X_{s}\right)$ and $v\left(X_{s}\right)$ for the zero chemical potential problem obtained by solving the non-stationary system with a standard phase initial state (dashed lines). We used Dirichelet boundary conditions $m(0)=m_{s}, \varepsilon(0)=\varepsilon_{s}, m(1)=m_{f}, \varepsilon(1)=\varepsilon_{f}$ on the finite interval $[0,1]$, at the coexistence pressure for $a=0.5, b=1, \alpha=100, k_{1}=k_{2}=k_{3}=10^{-3}$. Profiles at times $t=2 \times 10^{-7}, t=7.5 \times 10^{-6}, t=0.0100105, t=0.7930105, t=11.10100105, t=$ 1367.10100105 in lexicographic order. The gray lines represent stationary profiles. 

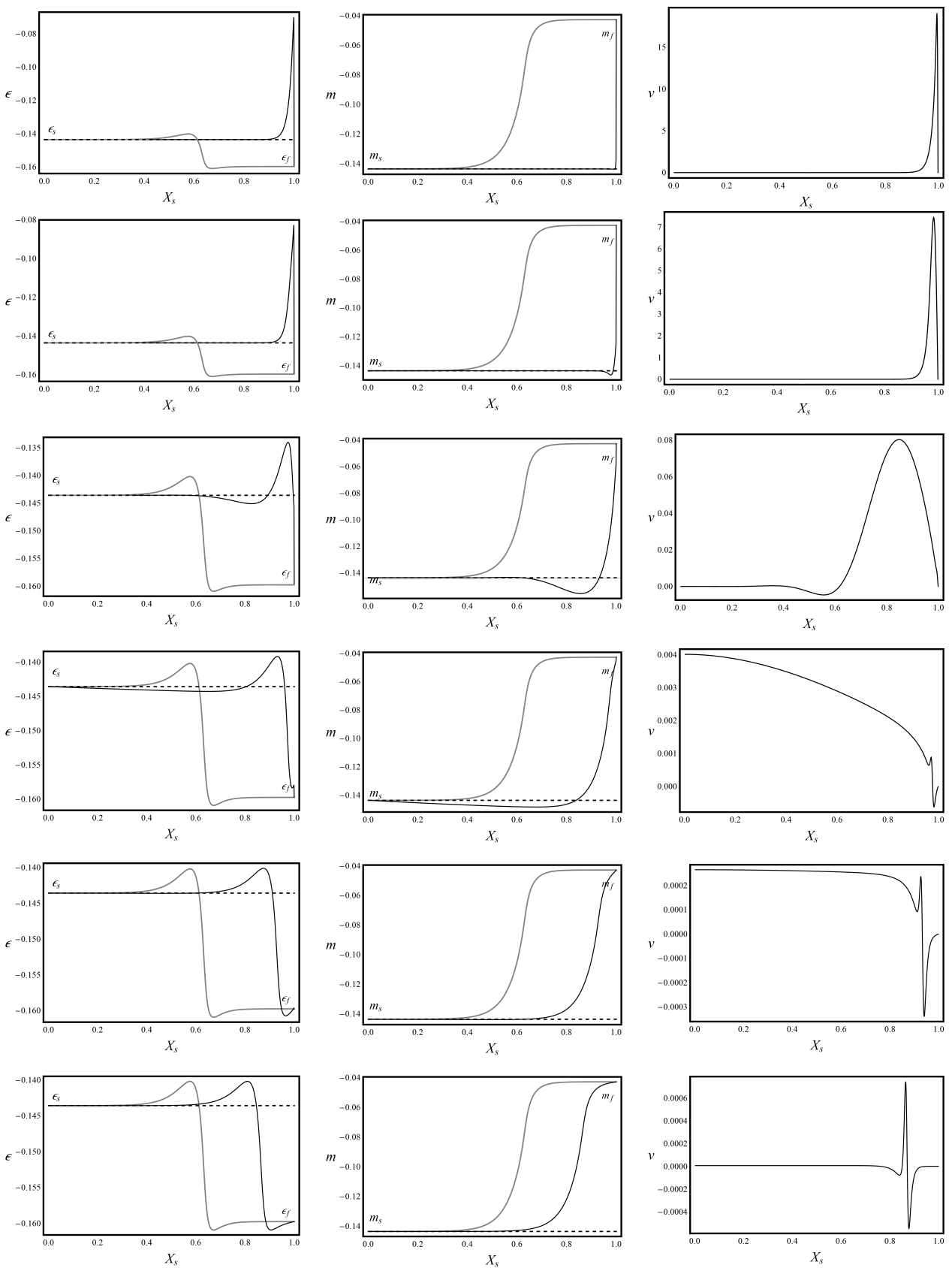

Figure 4.5: The same as in figure 4.4 but with the one side impermeability condition. 

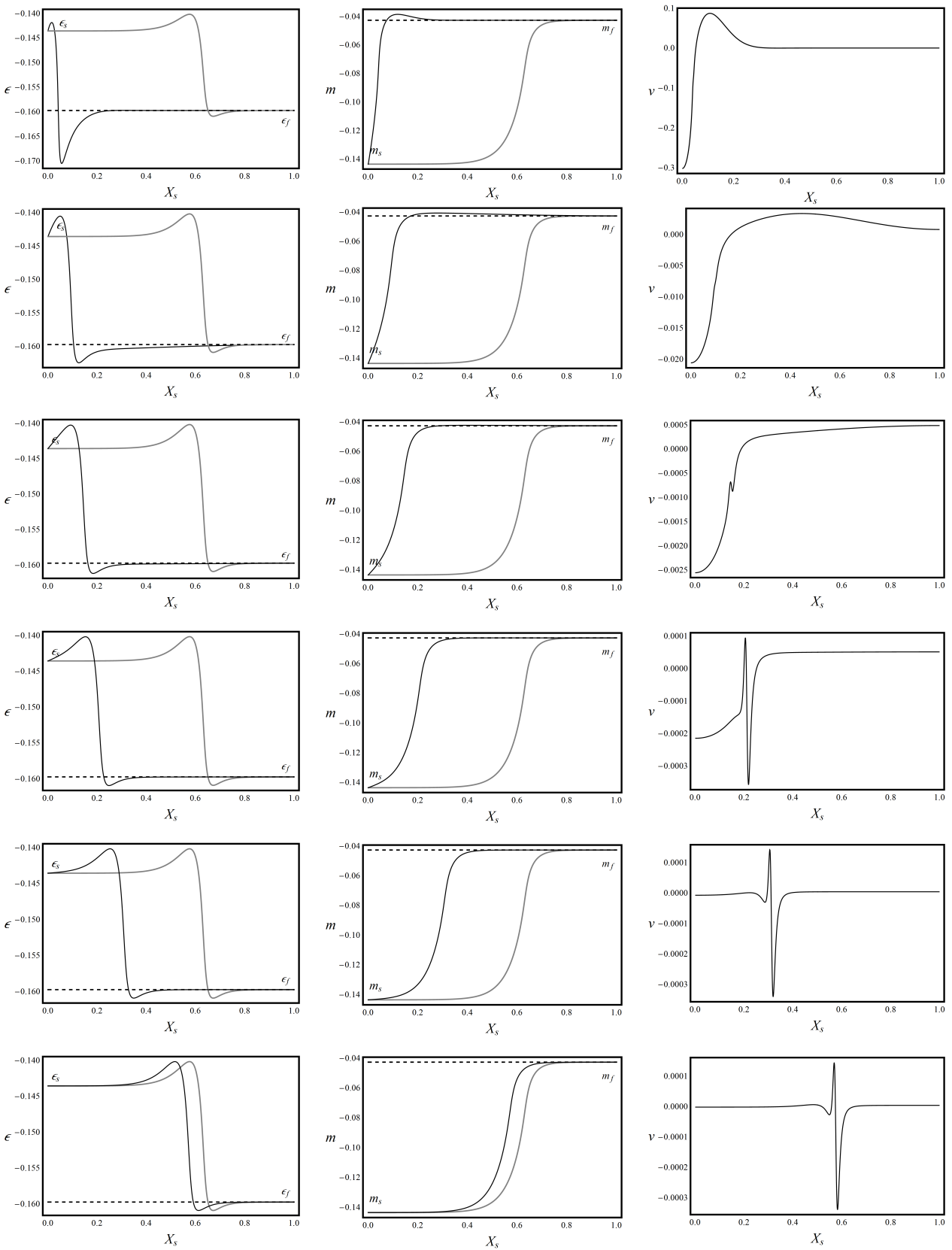

Figure 4.6: Profiles (black lines) $\varepsilon\left(X_{s}, t\right), m\left(X_{s}\right)$ and $v\left(X_{s}\right)$ for the zero chemical potential problem obtained by solving the non-stationary system with a fluid phase initial state (dashed lines). We used Dirichelet boundary conditions $m(0)=$ $m_{s}, \varepsilon(0)=\varepsilon_{s}, m(1)=m_{f}, \varepsilon(1)=\varepsilon_{f}$ on the finite interval [0,1], at the coexistence pressure for $a=0.5, b=1, \alpha=100, k_{1}=k_{2}=k_{3}=10^{-3}$. Profiles at times $t=0.004, t=0.08, t=0.8, t=8, t=83, t=333$ in lexicographic order. The gray lines represent stationary profiles. 

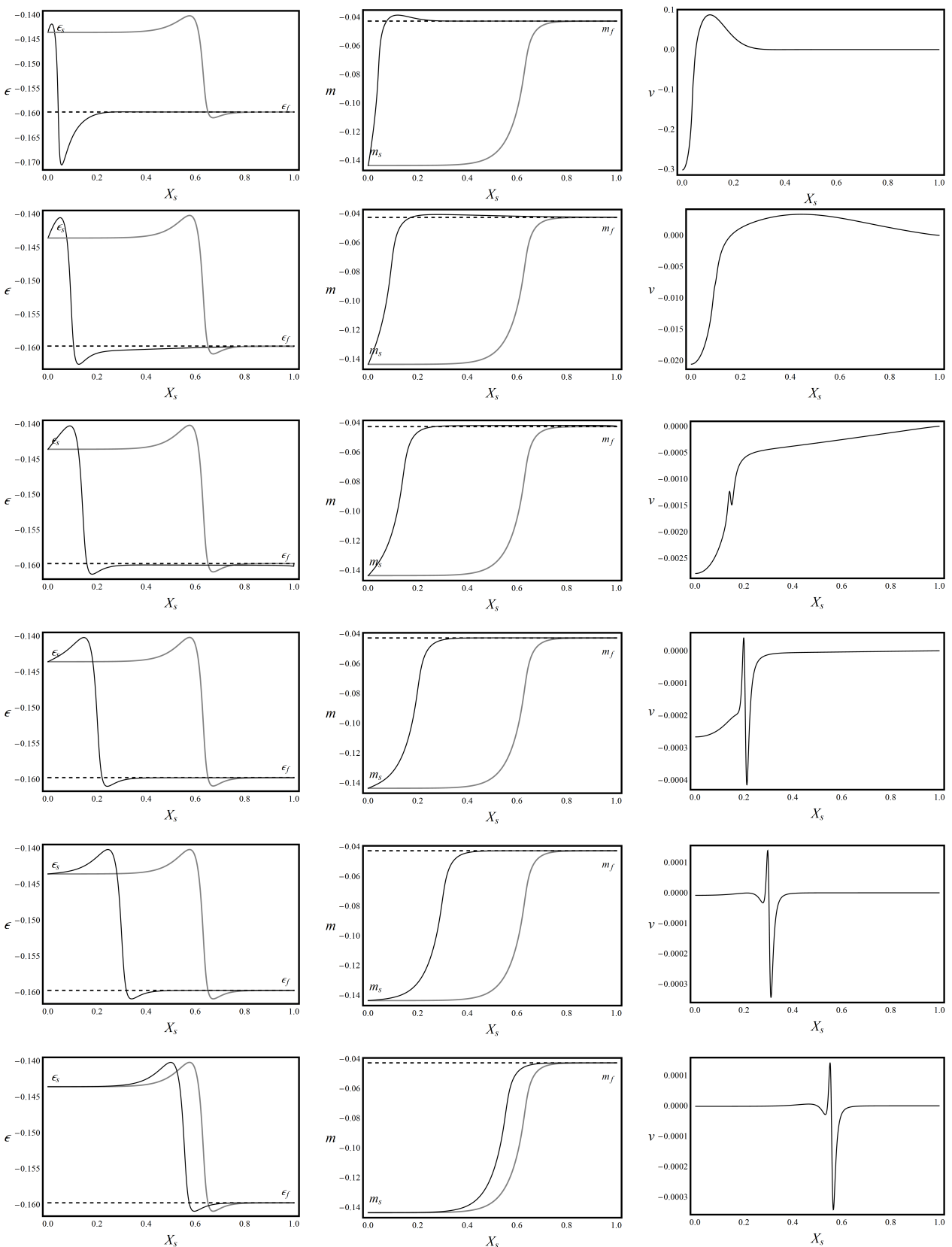

Figure 4.7: The same as in figure 4.6 but with the one side impermeability condition. 